\title{
Terapia de sustitución hormonal
}

\author{
Fabio Sánchez Escobar*
}

\begin{abstract}
RESUMEN: Debido a la mayor expectativa de vida, la mujer invierte una tercera parte en la menopausia, fenómeno que aunque fisiológico es una endocrinopatía que origina efectos catastróficos, por tal razón es necesario administrarle terapia de sustitución hormonal. En el presente artículo se hace un recuento de los riesgos beneficios, debido a la terapia hormonal y en especial en lo relativo al cáncer de mama y de endometrio. Además se describe la farmacología de las diferentes hormonas utilizadas, la dosis y las vías de administración, como también los efectos a nivel sistémicos. Igualmente se describe la terapia complementaria que se utiliza en la menopausia con respecto al calcio e indicaciones sobre el ejercicio.
\end{abstract}

PALABRAS CLAVES: Menopausia, Terapia de Sustitución Hormonal, Estrogenoterapia, Calcio, Ejercicio.

SUMMARY: Because of a longer life expectancy, women spend around a third part of their lifetime in the menopause stage, a physiological phenomenon which corresponds to an endocrinopathy with some catastrophic effects. For this reason it is necessary to supply a hormone substitution therapy in this paper the risk benefits of the hormone therapy and particularly those related with the breast, and endometrial cancer are described. In addition the pharmacology of the different hormones, doses, administration routes and systemic side effects are presented.

Similarly a calcium complementary therapy and exercise related indication are described.

KEY WORDS: Menopause, Hormone Substitution Therapy, Estrogen-therapy, Calcium, Exercise.

La terapia de sustitución o reemplazo hormonal consiste en administrar medicamentos a una paciente con el fin de suplir y alcanzar la dosis fisiológica de hormonas, tal como se actuaría en el caso de hipotiroidismo, enfermedad de Addison, hipopituitarismo o hipogonadismo. Por lo anterior, es importante tener en cuenta, aunque la mujer durante la vida reproductiva raras veces necesita terapia de sustitución estrogénica, la existencia de enfermedades asociadas al hipoestrogenismo y de esta manera poder iniciar una terapia oportuna que impida la aparición de los efectos secundarios a éste; tales efectos son semejantes a los que se presentan durante la menopausia.

Los trastornos ginecológicos que se acompañan de hipoestrogenismo son: 1) el hipogonadismo hipergonadotropo, como en los casos de la disgenesia gonadal síndrome de Tumer, disgenesia gonadal pura, síndrome de Swyer isocromía de brazos largos, entre otros-, la menopausia prematura y la menopausia iatrogénica; y 2) el hipogonadismo hipogonadotropo, como en los casos de síndrome de Kallman, la anorexia nerviosa, la bulimia, la menorrea por estrés o por ejercicio, el síndrome de Sheehan, la hiperprolactinemia idiopática o tumoral, y el hipotiroidismo. Muchas de esas enfermedades son de causa adquirida y pocas son de origen congénito.

En todas las anteriores enfermedades el factor común es el hipoestrogenismo de las pacientes, las cuales tienen

* Profesor titular. Departamento de Obstetricia y Ginecología. Facultad de Medicina. Universidad de Antioquia concentraciones séricas de estradiol por debajo de los 30 $\mathrm{pg} / \mathrm{ml}$, lo cual amerita una terapia de sustitución. Sin embargo, cuando se habla de terapia de sustitución estrogénica siempre se piensa en menopausia y se ignoran muchos de los trastornos antes mencionados, en los cuales las pacientes presentan los mismos síntomas y signos que en la menopausia; además, al igual que ésta dichas alteraciones hormonales deben ser tratadas profilácticamente para evitar graves consecuencias.

\section{Menopausia}

La menopausia es un síndrome cuya frecuencia se ha incrementado descle mediados del siglo XX debido al aumento de la expectativa de vida, pues a principios del siglo esta era sólo de 45 años, por lo cual muy pocas mujeres llegaban a la menopausia, mientras que actualmente la expectativa de vida es de 75 años en los países desarrollados y 68 en los que están en vía de desarrollo, hecho que favorece que un número mayor de mujeres lleguen a la menopausia y sufran los trastornos causados por la privación estrogénica. Por lo anterior se puede decir que la mujer vive un tercio de su vida sometida a los riesgos originados por la privación estrogénica.

En el estudio de la menopausia se han descrito síntomas y signos que según el tiempo de aparición pueden ser: 1) a corto plazo tales como las oleadas de calor, el insomnio, la fatigabilidad, la irritabilidad y la depresión; 2) a mediano plazo como la atrofia de la vulva, la vagina, 
la uretra y trígono, la mama y la piel; y 3) a largo plazo la osteoporosis y la arterioesclerosis. Todos los anteriores signos y síntomas son consecuencia de la pérdida estrogénica y justifican su profilaxis.

\section{Síntomas a corto plazo}

Los síntomas a corto plazo afectan cerca del $85 \%$ de las mujeres menopáusicas, pero sólo entre un 10 y un $15 \%$ de ellas los presentan tan severos que sientan la necesidad de consultar al médico. La consulta se hace principalmente por las oleadas de calor, las cuales son el síntoma más preponderante, pues cuando se presentan en las horas de la noche, hacen que la paciente tenga sueño superficial e insomnio; por ello cuando la mujer se levanta siente fatiga, adinamia, pocos deseos de trabajar, pérdida de la memoria y de la concentración, además expresa cambios de comportamiento, ansiedad, irritabilidad y depresión.

La situación antes descrita es una de las mayores causas de consulta médica; sin embargo, debido a la vaguedad de los síntomas, muchas pacientes consultan al psicólogo o al psiquiatra, viven relaciones difíciles con su familia y con su pareja porque no conocen la verdadera causa de su trastorno.

Desgraciadamente, la paciente en estado de menopausia tiene un alto grado de tolerancia a tales síntomas, por lo cual se abstiene de consultar, en tanto su familia no tiene la capacidad de entender el cambio de su comportamiento y, en muchas oportunidades, los hijos abandonan el hogar, se propicia la separación matrimonial, con el consiguiente aislamiento, sometimiento a tratamientos psiquiátricos $\mathrm{o}$, lo que es peor, reclusión en un hospital mental.

\section{Síntomas a mediano plazo}

Los síntomas a mediano plazo ocurren a través del tiempo como consecuencia de la atrofia del sistema genital, del urinario, y de la piel y mucosas, hechos que son entendidos por la paciente como procesos normales al envejecer; por ello, ésta sólo consulta por vaginitis, dispareunia, pérdida de la libido, polaquiuria, nicturia, disuria, sequedad de la piel y mucosas, y caída del cabello, síntomas que en conjunto se interpretan como el resultado de la pérdida estrogénica.

Puede suceder que el médico no haga el diagnóstico de menopausia por no interrogar ni examinar adecuadamente a la paciente, y se limite a tratarla sintomáticamente sin obtener, como es lógico, ninguna mejoría.

\section{Síntomas a largo plazo}

Los síntomas a largo plazo se presentan debido a que dos sistemas son afectados por la pérdida estrogénica: el cardiovascular y el óseo. En el primero se afectan las lipoproteínas y se presenta disminución de la HDL y aumento de la LDL, la VLDL y el colesterol total; este fenómeno posteriormente desencadena en la paciente la arterioesclerosis con todas sus consecuencias de hipertensión, angina de pecho, infarto cardíaco, enfermedad cerebrovascular y muerte.

Con relación al sistema óseo, una vez sucede la pérdida estrogénica se presenta una pérdida acelerada de la masa ósea de 3 al $6 \%$ anual en los primeros tres años, y del 1 al $3 \%$ anual durante el resto del tiempo, hasta llegar a los 75 años. Cuando las pacientes han perdido cerca del $25 \%$ del hueso cortical y un $50 \%$ del trabecular se quejan de artralgias que en ocasiones se confunden con artritis reumatoidea o se puede agravar la enfermedad y provocar una mayor inmovilización y, por ende, mayor osteoporosis hasta presentarse fracturas patológicas en la columna dorsolumbar, donde los aplastamientos vertebrales son frecuentes, en el cuello del fémur y en la parte distal del radio, huesos que tienen un alto contenido de hueso trabecular y, por ellos son más frágiles. Así, el 75\% de las fracturas patológicas se presentan en mujeres con más de 45 años de edad, una de cuatro mujeres mayores de ochenta años han sufrido de una fractura patológica y el $80 \%$ de las fracturas de cuello de fémur ocurre en mujeres osteoporóticas. Finalmente, estas pacientes aumentan progresivamente de peso debido a la mayor concentración de grasa corporal, (1) y comienzan una vida más sedentaria con la inmovilización y osteoporosis.

El anterior cuadro clínico es la consecuencia de la pérdida estrogénica, por tanto es la motivación para que el médico eduque y motive a su paciente con el fin de que cambie su sistema de vida y siga un terapia profiláctica dirigida a evitar estos fenómenos. De esta manera, aquél le asegura a ella una vida útil, productiva y carente de tantas complicaciones.

\section{Terapia de sustitución estrogénica: relación entre riesgos y beneficios}

Hay mucha controversia con respecto a la terapia de sustitución estrógenica, en la menopausia, la cual es un fenómeno que se ha agravado debido al mayor aumento de la población de mujeres menopáusicas. Así, cada vez se comparte la duda sobre si toda paciente con insuficiencia endocrina, sea en la glándula hipófisis, la tiroides o la suprarrenal, reciben tratamiento de suplencia, porque en la insuficiencia ovárica, fisiológica o adquirida, no se administra ningún tratamiento.

Por otra parte, el debate mundial sobre la relación entre riesgos y beneficios de la terapia hormonal se basa en que si bien es cierto que existen riesgos, tal como el carcinoma de endometrio y el de mama, también lo es que se han obtenido beneficios, como por ejemplo menor mortalidad por infarto del miocardio, por accidente cerebrovascular e igualmente menor frecuencia en fractura de fémur (2-3). Lo anterior se demuestra en la tabla 1, en la cual los riesgos constituyen el número de muertes por cien mil mujeres y los beneficios el número de muertes evitables con la terapia por cien mil mujeres.

La tabla 1 muestra una relación de 5,6 veces de efecto benéficos respecto a las prevenciones de muertes en pacientes con terapia hormonal que tienen útero y de 9,4 veces en las que no lo tienen. Este pronóstico puede mejorarse, aún más, si se le mezclan progestágenos de la familia de la 17 Hidroxiprogesterona a la terapia estrogénica, lo cual se puede apreciar en la tabla 2.

En la tabla 2 se observa una relación 6,4 veces de efectos benéficos con respecto a la disminución en el número de muertes en pacientes con útero y sin él al disminuir la frecuencia del cáncer de endometrio con el 
Tabla 1

Relación entre riesgos y beneficios de la terapia estrogénica, medida por cien mil mujeres

\begin{tabular}{|lcc|}
\hline Enfermedad $^{\mathrm{a}}$ & Riesgo $^{\mathrm{b}}$ & Beneficio $^{\mathrm{c}}$ \\
\hline Cáncer de mama & 39 & \\
Cáncer de endometrio & 26 & \\
Infarto del miocardio & & 284 \\
Accidente cerebrovascular & & 49 \\
Fractura de fémur & & 33 \\
\hline
\end{tabular}

- La relación entre el riesgo y el beneficio de la terapia estrogénica en las mujeres con útero es 5,6 y en las mujeres sin útero es 9,4 .

b El total de las mujeres atribuidas en la terapia estrogénica es 65 por cada cien mil mujeres

El total de las muertes evitadas por la terapia estrogénica es 366 por cada cien mil mujeres.

progestágeno. Sin embargo, llama la atención la disminución de los efectos benéficos en los accidentes cerebrovasculares, hecho éste esperado si se prescriben progestágenos derivados de los 19 nor-esteroides, ya que éstos alteran las lipoproteínas; este fenómeno no se presenta con el empleo de la medroxiprogesterona, en cuyo caso la prevención de muertes por infarto del miocardio y por accidente cerebrovascular sigue siendo igual que en la terapia estrogénica, como lo muestra la tabla 1, dicho efecto benéfico es de 9,4 .

\section{Riesgos}

Los riesgos que se presentan durante la terapia estrogénica son el carcinoma de endometrio y el carcinoma de mama.

Tabla 2

Relación entre riesgos y beneficios de la terapia combinada de estrógenos y progestágenos, medida por cada cien mil mujeres

\begin{tabular}{|lrr|}
\hline Enfermedad $^{\mathrm{a}}$ & riesgo $^{\mathrm{b}}$ & beneficio $^{\mathrm{c}}$ \\
\hline Cáncer de mama & 39 & \\
Cáncer de endometrio & 0 & \\
Infarto del miocardio & & 184 \\
Accidente cerebrovascular & & 32 \\
Fractura de fémur & 33 \\
\hline
\end{tabular}

a La relación entre el riesgo y el beneficio de la terapia combinada es de 6,4 y es igual para mujeres con útero o sin él.

- El total de muertes atribuidas a la terapia combinada es de 39 por cada cien mil mujeres.

El total de muertes evitadas por la terapia combinada es 249 por cada cien mil mujeres.

\section{Carcinoma de endometrio}

El carcinoma de endometrio es uno de los riesgos que tiene la acción estrogénica persistente, tal como ocurre en el síndrome de ovario androgénico y en la terapia de sustitución por estrógenos. La frecuencia con la cual se presenta dicho carcinoma se eleva de 245,5 por cada cien mil mujeres, entre la población general, a una proporción dos a cuatro veces mayor entre las pacientes con sustitución estrogénica; como si fuere poco, esta frecuencia se aumenta en forma proporcional al tiempo de ingesta y, en espacial, después de cinco años.

Secundariamente a la terapia se ha descrito un carcinoma de endometrio poco invasor y bien diferenciado; no se sabe si esto se debe a una característica de la enfermedad, a un diagnóstico precoz o a hiperplasias atípicas mal diagnosticadas como carcinoma. El promedio de sobrevida de las pacientes en mención es mayor a los cinco años, 92\%, comparado con el promedio de sobrevida de las mujeres que presentan carcinoma de endometrio y que no han consumido estrógenos, $68 \%$.

Este efecto negativo de la terapia hormonal se ha contrarrestado con el empleo de progestágenos derivados de la 17 Hidroxiprogesterona, en dosis de $10 \mathrm{mg}$ durante diez a catorce días o $2,5 \mathrm{mg}$ diariamente por tiempo indefinido, terapia que ha disminuido el riesgo de carcinoma de endometrio a proporciones iguales a las de la población general (4). Los posible mecanismos que se aducen para explicar este efecto benéfico de los progestágenos son dos: (5) 1) bloquean la síntesis de receptores estrogénicos hasta agotarlos y 2) aumentan la síntesis de la enzima 17ß-deshidrogenasa, la cual transforma el estradiol en estrona, hormona con menor actividad biológica. Bien sea por uno o por otro efecto, la proliferación endometrial es menor, por esta razón se puede encontrar desde endometrios proliferativos iniciales hasta verdaderas atrofias.

Thom describió frecuencias de hiperplasia de endometrio del $15 \%$ en pacientes con terapia estrogénica, del $2 \%$ cuando se le agregan progestágenos por cinco a diez días, y de $0 \%$ cuando se administran por trece días (6). Igualmente, Studd encontró que la incidencia de hiperplasia endometrial es de $15 \%$ cuando se utilizan estrogénos conjugados a la dosis de $1,25 \mathrm{mg}, 7 \%$ cuando se dan $0,625 \mathrm{mg}$, desciende al $2 \%$ con progestágenos por cinco a diez días y no existe cuando se dosifican por trece días o más (7).

Por otra parte, Gambrell afirma que la frecuencia de carcinoma endometrial desciende de 390 por cada cien mil usuarias de estrogénos continuos a 49 por cien mil cuando se agregan progestágenos, además, éste indica que la incidencia en los Estados Unidos es de 21.3 por cada cien mil mujeres blancas y de 13.1 en las de raza negra (4). En tanto, otro autor dice que la mortalidad por carcinoma de endometrio desciende de veintiséis por cada cien mil usuarias de estrógenos a cero cuando se agregan progestágenos (2).

Un dilema que frecuentemente se presenta es si darle la terapia estrogénica a una paciente tratada por carcinoma de un endometrio y cruda. Este se clarifica en un estudio al azar efectuado en 221 pacientes tratados con cáncer de endometrio estadío 1 , de las cuales 47 recibieron terapia estrogénica y 147 no lo hicieron, hubo un $2,1 \%$ de recidivas en el grupo tratado con terapia hormonal y $14,9 \%$ en el no tratado, un paciente murió del primer grupo y veintiséis del segundo, por esta razón parece que la terapia estrogénica tiene un efecto protector sobre las que la utilizan con respecto a las que no lo hacen; además 
dicho efecto benéfico es mayor con relación a la osteoporosis y los accidentes cardiovasculares (8). Este dilema es importante para futuras investigaciones.

\section{Cáncer de mama}

El efecto de la terapia estrogénica sobre la mayor incidencia del carcinoma de mama es un tema muy debatido, se han hecho muchos estudios -de tipo casos y controles, cohortes y, últimamente, estudios de metaanálisis- pero, aún no se ha obtenido un criterio claro, ya que algunos autores hablan de efecto benéfico de la terapia hormonal mientras que otros indican los riesgos de ésta.

Por ejemplo, Steimberg (9) en un estudio expresa el riesgo relativo de 1,3 que se desarrolle esta enfermedad en usuarias de terapia estrogénica durante quince años, esto equivale a un $0,3 \%$ de riesgo mayor en éstas que en la población general; por esta razón se estima que la terapia estrogénica puede agregar anualmente 39 muertes por cada cien mil mujeres entre 65 y 74 años a las 102 muertes por cada cien mil debidas al cáncer de mama (2).

Por otra parte, en un estudio de metaanálisis de Sillero-Arenas y colaboradores (10) se encuentra un riesgo relativo de 1,63 en pacientes con terapia estrogénica y de 1,6 en pacientes con terapia de estrógenos y progestágenos; por ello se concluye que la terapia hormonal puede tener un papel en el riesgo de cáncer de mama, pero en una frecuencia que no es significativa, pues, si este riesgo se analiza con relación a los beneficios se encuentra despreciable.

\section{Vías de administración}

Existen varias vías de administración de estrógeno, las cuales tienen indicaciones específicas y dependen de las diversas presentaciones de la hormona (11)(Véase tabla 3)

\section{Vía oral}

La vía oral es la más utilizada universalmente tanto para los estrógenos conjugados, como el sulfato de estrona que se presenta en concentraciones de 0,625 y $1,25 \mathrm{mg}$; como para los sintéticos entre los cuales se encuentra el valerianato de estradiol en comprimidos de $2 \mathrm{mg}$.

La droga al ser ingerida sufre una 17 reducción de estradiol a estrona en la mucosa duodenal, además, los estrógenos orales provocan concentración muy elevada en la circulación enterohepática la cual desencadena en algunas situaciones la alteración del funcionamiento hepático, por este motivo se debe proscribir esta vía en pacientes con patología hepática y en hipertensos. $\mathrm{La}$ tercera parte de los estrógenos que llegan al hígado se transforman a 3 glucoronido de estrona, compuesto que es eliminado por la bilis y la orina, y sólo las dos terceras partes son activas, razón por la cual los estrógenos orales deben administrarse en mayor concentración que los parentenales, y la relación entre estradiol y estrona es invertida con relación a la mujer en edad media. Dicha inversión no es importante, pues, al final ambos compuestos tienen un efecto benéfico sobre la sintomatología de la menopausia.

La absorción de los estrógenos orales se hace en picos, una hora después de la ingesta, y la concentración que éstos alcanzan cae lentamente hacia las veinticuatro horas. Cuando los estrógenos orales pasan a través del hígado en concentraciones elevadas causan aumento de la globulina transportada de los esteroides sexuales SHBG-, la globulina transportadora de cortisol y progesterona -trascortina-, y el sustrato de renina; además, éstos aumentan las lipoproteínas de alta densidad HDL- y disminuyen las de baja densidad -VLDL- y la antitrombina III; este último efecto producido en el hígado es más potente cuando se administran estrógenos sintéticos, tales como etinil estradiol y dietilestilbestrol, los cuales no son utilizados en la terapia de sustitución precisamente por el riesgo que presentan al asociarse con hipertensión y tromboembolismo.

En raras ocasiones los estrógenos orales pueden causar náuseas, vómitos y epigastralgia, por lo cual es necesario suspender el tratamiento o cambiar la vía de administración. Estas drogas pueden perder su actividad biológica en pacientes que ingieren difenil hidantoína.

\section{Vía local}

La vía local se utiliza para la aplicación de los estrógenos cuya presentación es en cremas para aplicación por vía vaginal o en pesarios de liberación continua; la primera de ellas es utilizada en Colombia.

La absorción vaginal es más rápida que la oral, pero se requiere una dosis mayor de hormona, pues, $1,25 \mathrm{mg}$ de estrógenos conjugados por vía vaginal equivale a

Tabla 3

Vías de administración de las diferentes presentaciones de estrógenos

\begin{tabular}{|c|c|c|}
\hline Vía ${ }^{a}$ & Preparación & Nombre comercial \\
\hline Oral & Tabletas & $\begin{array}{l}\text { Proginova }{ }^{\circledR} 2 \mathrm{mg}^{\mathrm{b}} \text {, Ayerogen }(0,625 \mathrm{y} \\
1,25 \mathrm{mg}^{\mathrm{c}}\end{array}$ \\
\hline Local & $\begin{array}{l}\text { Crema vaginal, } \\
\text { pesarios o anillos vaginales }\end{array}$ & \\
\hline Parenteral & $\begin{array}{l}\text { Cremas percutáneas } \\
\text { implantes subcutáneos } \\
\text { o parches trasdérmicos }\end{array}$ & Estraderm $^{\mathrm{d}} 2$ y $4 \mathrm{mg}$ \\
\hline
\end{tabular}

\footnotetext{
a Otras vías de administración son la intravenosa, la intramuscular, la sublingual y la intranasal.

b El nombre genérico es valerianato de estradiol

c El nombre genérico es sulfato de estrona

d El nombre genérico es 17 Bestradiol
} 
0,625 mg por vía oral. Además, esta vía tiene la ventaja de que el estradiol no se metaboliza a estrona, por lo cual la relación entre estradiol y estrona es mayor de uno.

La absorción del estrógeno por vía vaginal es pobre durante la primera semana, pero mejora de la segunda en adelante como consecuencia del mayor trofismo y la mayor vascularización; además, esta absorción se aumenta cuando el estrógeno se disuelve en solución salina que cuando se hace en aceite.

También, los estrógenos pueden estar en anillos de silicona que se impregnan en estradiol, los cuales se aplican y extraen fácilmente; en la vagina éstos liberan cerca de 0,2 mg de estradiol en un día. Esta presentación no se encuentra disponible en Colombia.

\section{Vía Parenteral}

La vía parenteral se utiliza para la administración de estrógenos en el hidroalcohólico como rubefaciente, presentación que permite mejorar su absorción, aunque se realiza en forma lenta y en concentración constante. La aplicación percutánea del gel se hace en la parte inferior del abdomen, en una cantidad de $5 \mathrm{~g}$ que contienen $3 \mathrm{mg}$ de estradiol, el cual pasa a la circulación sistemática donde se degrada y queda una relación entre estradiol y estrona igual a 1,5 .

La aplicación percutánea del estrógeno se hace cuando están contenidos en parches de $3 \mathrm{~cm}$ de diámetro, en su interior está el estrógeno o valerianato de estradiol de 2 ó $4 \mathrm{mg}$ en solución alcohólica lo que permite su absorción. El parche es adherente y se aplica cada cuatro días en el abdomen, o en la región glútea de tal manera que su superficie le permite una fijación firme a la piel. El esquema aconsejado para la utilización del fármaco consiste en que la paciente se coloque un parche cada cuatro días por tres semanas y deje una semana de descanso; además, durante el tiempo de aplicación del parche, aquella debe ingerir $25 \mathrm{mg}$ de medroxiprogesterona.

El parche se denomina de acuerdo con los microgramos de estrógeno que libera por día, por ejemplo estraderm 25 , Estraderm ${ }^{\circledR} 50$ y Estraderm ${ }^{\circledR} 100$; liberan 25,50 o 100 ig; los cuales permiten alcanzar concentraciones plasmáticas de estradiol de 23,39 y $74 \mathrm{pg} / \mathrm{ml}$ respectivamente.

La aplicación percutánea de estradiol hace disminuir los niveles de LDL, y elevar la HDL, y no tiene efecto sobre la síntesis de sustrato de renina por lo cual se puede utilizar en pacientes con hipertensión arterial (12-13).

La vía parenteral también se utiliza para la administración de estrógenos en forma de implantes, éstos son barras impregnadas con 50 o $100 \mathrm{mg}$ de estradiol y deben ser aplicadas por el médico debajo de la piel y con anestesia local. Dicho fármaco inicia rápidamente la liberación del estrógeno, alcanza la liberación máxima en dos o tres meses y tiene una duración no mayor de seis meses. La concentración plasmática de la hormona es tal que la relación entre estradiol y estrona se encuentran por encima de 1,0; en ocasiones los estrógenos se implantan combinados con $100 \mathrm{mg}$ de testosterona, con el fin de evitar la hepatotoxicidad de la metiltestosterona cuando se emplea por vía oral.

Además de las vías para la administración de estrógenos anteriormente detalladas, existen otras que, aunque se emplean en la terapéutica médica, no se utilizan en la terapia de sustitución hormonal porque son hormonas de acción prolongada y en la terapia de sustitución es necesario fármacos de acción corta y aplicación diaria. Tal es el caso de las vías intramuscular e intravenosa; así, preparados como el hexahidroxibenzoato de estradiol conocido comercialmente como Menodin RetardR ${ }^{\circledR}$ de $5 \mathrm{mg}$ y de acción retardada, y el valerianato de estradiol, el cual comercialmente es el Progynon Depot ${ }^{\circledR}$ de $10 \mathrm{mg}$ se aplican por vía intramuscular cada quince a treinta días. Los estrógenos conjugados como el Ayerogen ${ }^{\circledR}$ de $25 \mathrm{mg}$ son de acción corta, por lo cual se utilizan en caso de hemorragia uterina disfuncional y no se usan en la terapia de sustitución.

La vía sublingual y, especialmente, la intranasal están en investigación y se utilizan no sólo para estrógenos sino también para aplicar gonadotropina coriónica humana, calcitonina y otras hormonas. Además, la vía subdérmica es útil para la administración de estrógenos en forma de microcristales de liberación lenta o pelettes, pero éstas aún no se venden en Colombia.

\section{Vía de elección}

La gran variedad de vías de aplicación del estrógeno permite al médico seleccionar, con la paciente, la vía de prescripción de acuerdo con los beneficios que se quieren obtener y con los riesgos que se presentan. Así, la vía oral es la más universal y la más tolerable, pero se debe evitar su utilización en pacientes que tienen intolerancia a la droga, que padecen enfermedades hepáticas, o que son hipertensas no controladas. La vía parenteral se utiliza en pacientes que olvidan ingerir la droga, tienen el antecedente de enfermedad tromboembólica reciente, o presentan contraindicaciones para el uso de la droga por vía oral. La vía vaginal se utiliza en los casos en los cuales la atrofia vaginal y el síndrome uretral no han mejorado con otra vía de aplicación.

\section{Esquemas de prescripción}

Los esquemas para terapia de sustitución estrogénica que existen son múltiples y las razones para utilizar uno u otro esquema también lo son. A continuación se detallan algunos esquemas para la administración de estrógenos.

\section{Esquema para la administración de estrógenos por vía oral}

La administración por vía oral se realiza siguiendo uno de los siguientes esquemas:

1. El esquema de dosis continua consiste en la administración de estrógenos diariamente en forma indefinida; así, se prescribe valerianato de estradiol de $2 \mathrm{mg}$ o sulfato de estrona de $0,625 \mathrm{mg}$ más progestágenos tipo medroxiprogesterona de $2,5 \mathrm{mg}$ por día indefinidamente.

2. El esquema de dosis cíclica consiste en la administración de hormonas de las dos formas siguientes: 1) la paciente ingiere estrógenos diariamente por tres semanas, en la última de ellas combina estrógenos con progestágenos y descansa una semana para volver a comenzar el ciclo; o 2) la paciente ingiere estrógenos diaria e indefinidamente y progestágenos durante los primeros diez o catorce días de cada mes. 
Existen otros esquemas de administración cíclica combinada en los cuales se mezclan ambas hormonas, tal como sucede con el climene que consiste en catorce comprimidos con valerianato de estradiol de $2 \mathrm{mg}$ y siete comprimidos con valerianato de estradiol de $2 \mathrm{mg}$ más acetato de ciproterona de $1 \mathrm{mg}$.

Esquema para la administración de estrógenos por vía parenteral

Los estrógenos en parches de 25 o $50 \mathrm{mg}$ combinados con medroxiprogesterona de $2,5 \mathrm{mg}$ por día y por vía oral, se aplican cada cuatro días durante tres semanas y se deja una semana de descanso o se administra en forma continua.

Esquema para la administración de estrógenos por vía vaginal

La crema de sulfato de estrona se aplica de tal forma que la dosis sea el doble de la que se usa por vía oral, esto es 1,25 de sulfato de estrona diariamente, para alcanzar la concentración plasmática adecuada y se combina con progestágenos que la paciente debe ingerir en forma continua o cíclica.

\section{Farmacología}

Los estrógenos utilizados para la terapia de sustitución se clasifican en: naturales, semisintéticos, sintéticos $\mathrm{y}$ equinos.

\section{Estrógenos naturales}

Los estrógenos naturales son el estradiol, la estrona y el estriol. El primero es el más representativo de ellos y cuando se administra por vía oral es rápidamente metabolizado a estrona en la mucosa intestinal por la enzima 17 beta hidroxiesteroide deshidrogenasa. Para evitar este fenómeno se está utilizando el estradiol en partículas pequeñas, es decir estradiol micronizado, lo que le permite una absorción rápida en el intestino, sin ser metabolizado en tal altas proporciones.

La absorción oral y la actividad biológica de los estrógenos naturales puede ser aumentada en la forma de sal, tal como el valerianato de estradiol, que le permite a la hormona ser más estable. Otros compuestos hacen que el estradiol se libere lentamente cuando se libera en varias horas, mientras que el valerianato y el undecilato se demoran hasta veinte días.

\section{Estrógenos semisintéticos}

Los estrógenos semisintéticos son el etinil estradiol y el mestranol. Estos se originan de modificaciones en la estructura del estradiol tal como ocurre con el etinil estradiol, EE, el cual se forma al agregar un grupo etinil en el carbono 17 del estradiol; y con el mestranol, que se origina al agregar un grupo metilo, $\mathrm{CH}_{3}$, en el carbono tres del etinil estradiol. Gracias a estas modificaciones se obtiene un compuesto estrogénico cien veces más potente que el estradiol, debido a que dicho compuesto es resistente a la acción de la 17 beta hidroxiesteroide deshidrogenasa en la mucosa intestinal y a que no se une a la globulina transportadora de esteroides sexuales, se acumula en los tejidos y tiene lenta eliminación urinaria. Por lo anterior, estos compuestos se usan en microdosis en los anticonceptivos orales y no se utilizan en la terapia de sustitución.

\section{Estrógenos disponibles en Colombia}

La tabla que se presenta a continuación muestra los preparados de estrógenos que se encuentran disponibles en Colombia.

Tabla 4

Presentación y dosis de los estrógenos disponibles en Colombia

\begin{tabular}{|llcl|}
\hline Estrógenos naturales & Nombre comercial & $\begin{array}{c}\text { Concentración } \\
\text { mg }\end{array}$ & $\begin{array}{c}\text { Vía de } \\
\text { administración }\end{array}$ \\
\hline 17 Beta estradiol & Estraderm TS 25® & 2 & Parenteral \\
& Estraderm TS 50® & 4 & Parenteral \\
Valerianato de & Progynova & Oral \\
estradiol & Progynon Depot ${ }^{\circledR}$ & 10 & Parenteral \\
Hexahidroxibenzoato de estradiol & Menodin Retard® & 5 & Parenteral \\
Sulfato de estrona & Ayerogen ${ }^{\circledR}$ & 0,625 & Oral y venosa \\
16 alfahidroxiestrona & Novergon ${ }^{\circledR}$ & 1,25 & Parenteral \\
\hline
\end{tabular}

\section{Terapia de sustitución estrogénica combinada con progestágenos}

La terapia de sustitución estrogénica se combina con la administración de progestágenos, que son sustancias con un efecto semejante a la progesterona natural, pero son obtenidos artificialmente y se clasifican de acuerdo con su origen en progestágenos naturales y sintéticos, tal como lo muestra la tabla 5 .

\section{Compuestos naturales}

La progesterona es la hormona natural por excelencia, pero al ser administrada por vía oral es metabolizada rápidamente en el tubo digestivo, por lo cual sólo se puede prescribir por vía parenteral cada doce horas a la dosis de 50 o de $100 \mathrm{mg}$ vía intramuscular. La progesterona micronizada se utiliza actualmente, pues, gracias a su presentación en micras es menor su degradación y 
Tabla 5

Clasificación de los progestágenos

\begin{tabular}{|c|c|c|}
\hline Origen & Compuesto & Nombre comercial \\
\hline $\begin{array}{l}\text { Natural } \\
\text { Sintético }\end{array}$ & Progesterona & Progesterona ${ }^{\circledR}$ \\
\hline $\begin{array}{l}\text { Sintético } \\
17 \text { Hidroxiprogesterona }\end{array}$ & $\begin{array}{l}\text { Acetato de medroxipro- } \\
\text { gesterona } \\
\text { Acetato de clormadinona } \\
\text { Meprogestone } \\
\text { Caproato de } 17 \text { Hidroxi- } \\
\text { progesterona }\end{array}$ & $\begin{array}{l}\text { Provera }{ }^{\circledR} 2,5 ; 5 y 10 \mathrm{mg} \\
\text { Depoprovera }{ }^{\circledR} 50 \mathrm{mg} \cdot \mathrm{ml} \\
\text { Clormadinona }^{\circledR} \\
\text { Proluton-Depot }{ }^{\circledR} 2 \\
50 \text { y } 500\end{array}$ \\
\hline \multirow[t]{2}{*}{19 Norprogesterona } & $\begin{array}{l}\text { Acetato de megestrol } \\
\text { Promegestone } \\
\text { Demegestrone }\end{array}$ & Megace ${ }^{\circledR} 20,40$ y 160 \\
\hline & Caproato de gestonorona & Primostat $® 200 \mathrm{mg}$ \\
\hline \multirow[t]{2}{*}{19 Nortestosterona } & $\begin{array}{l}\text { Acetato de noretindrona } \\
\text { Enanato de noretisterona }\end{array}$ & $\begin{array}{l}\text { Primolut } \text { Nor }^{\circledR} 5 \text { y } 10 \\
\text { Noristerat }{ }^{\circledR} \\
20 \text { intramuscular }\end{array}$ \\
\hline & $\begin{array}{l}\text { Noretinodrel } \\
\text { Linestrenol } \\
\text { Diacetato de etinodiol } \\
\text { L-norgestrel }\end{array}$ & $\begin{array}{l}\text { Desogestrel }{ }^{\circledR} \\
\text { Norgestimato }{ }^{\circledR} \\
\text { Gestadeno }\end{array}$ \\
\hline
\end{tabular}

permite su absorción rápida a través de la mucosa intestinal.

Además, la progesterona se ha utilizado por vía vaginal o rectal en forma de óvulos de 25 y $50 \mathrm{mg}$ cada ocho a doce horas pero esto se hace sólo en los casos de mujeres en el primer trimestre de embarazo y con diagnóstico de cuerpo lúteo insuficiente. Esta forma de administración no se utiliza en mujeres en menopausia.

\section{Compuestos sintéticos}

Múltiples compuestos sintéticos tienen efecto progestacional a pesar de tener origen diferente al de la progesterona natural. Muchos de ellos son derivados de la 19 Nortestosterona; éstos son muy potentes, por tanto se utilizan en dosis muy pequeñas por vía oral, y hacen parte de los anticonceptivos. Otros se derivan de la 19 Norprogesterona y de la 17 Hidroxiprogesterona, de ellos los últimos son los más utilizados.

Muchos de los progestágenos derivados de la 19 Nortestosterona tienen como efecto colateral la disminución de la HDL-C y el aumento de la LDL-C y la VLDLC, por lo cual no se utilizan en la terapia de sustitución; sin embargo, los nuevos compuestos progestacionales existentes en los anticonceptivos orales pueden ser de mucha utilidad y posiblemente no tienen dichos efectos secundarios; tal es el caso del gestadeno, el desogestrel y el norgestimato que son compuestos derivados del LNorgestrel.
Los progestágenos más utilizados en la terapia de sustitución son los derivados de la 17 Hidroxiprogesterona, tal como el acetato de medroxiprogesterona o provera que se presenta en comprimidos de 2,5;5 y $10 \mathrm{mg}$, pues, éstos no producen modificaciones en los niveles de las lipoproteínas. Dichos compuestos se utilizan a la dosis de $10 \mathrm{mg}$ por día durante diez a catorce días en mujeres que desean continuar mestruando o de $2,5 \mathrm{mg}$ por día indefinidamente cuando aquellas desean entrar en la amenorrea (14-15).

La potencia de los progestágenos se puede medir evaluando algunos parámetros de la siguiente manera:

1. La queratinización del epitelio vaginal indica el grado de actividad antiestrogénica; dicho grado es igual a uno para la progesterona y a 74 para el Norgestrel, por ello este último es el más potente.

2. La potencia androgénica se evalúa midiendo el crecimiento de la próstata en los animales, el resultado es cero para el acetato de medroxiprogesterona, 4,7 para el D/L Norgestrel y 9,4 para el Levonorgestrel, por ello este último es el más potente.

3. La potencia progestacional se mide por medio del test de Clauberg, el cual consiste en administrar el progestágeno que se está investigando a conejos con pesos entre 800 y $1000 \mathrm{~g}$ durante cinco días y por vía oral o subcutánea; el día sexto se sacrifican los conejos y se evalúa el grado de proliferación endometrial según la escala de PcPhail (Véase tabla 6) 
Tabla 6

Potencia de los progestágenos de acuerdo con el test de Clauberg

\begin{tabular}{|lcc|}
\hline Compuesto & Vía subcutánea & Vía oral \\
\hline Progesterona & 1 & 1 \\
17 acetato de & 1 & 1 \\
hidroxiporogesterona & 25 & 7,5 \\
Disprogesterona & 2,5 & 33 \\
Linestrenol & 2,5 & 33 \\
Noretrinodrel & 2,5 & 33 \\
Acetato de gestenorona & 2,5 & 100 \\
Dimetisterona & 7,5 & 100 \\
Diacetato de etinodiol & 12 & 100 \\
Noretisterona & 25 & 330 \\
Acetato de noretisterona & 66 & - \\
L-norgestrel & & \\
Acetato de & 75 & 333 \\
medroxiprogesterona & 75 & 333 \\
Acetato de clormadinona & 2 & 400 \\
Acetato de megestrol & 250 & 1000 \\
Acetato de ciproterona & & \\
\hline
\end{tabular}

\section{Terapia complementaria}

Las pacientes sometidas a terapia de sustitución necesitan ser estimuladas hacia un cambio de estilo de vida. Dicho cambio comprende: el cuidado en la administración de otros medicamentos, el seguimiento de una dieta adecuada, la realización de ejercicio, y una actitud positiva ante la vida, lo cual incrementa la autoestima y plantea un papel que la paciente desempeña en su familia y en la sociedad.

\section{Dieta}

Las pacientes sometidas a terapia de sustitución hormonal requieren una buena ingesta de calcio y una dieta bien balanceada para asegurar la actividad osteogénica, debido a que el $30 \%$ del hueso consta de matriz orgánica y el $70 \%$ de sales (16). Entre el 90 y el $95 \%$ de la matriz orgánica es colágeno, el estrógeno juega un papel importante en la síntesis de éste, y el 5\% de aquella es sustancia fundamental que está constituida por proteoglicanos, de los cuales el ácido hialurónico y el condrohitin sulfúrico son los elementos principales. El material orgánico está conformado por la hidroxiapatita, una sal que tiene iones de sodio, magnesio, carbonato y citrato; pero los componentes más importantes son los fosfatos y el calcio.

La mujer en la menopausia aumenta los requerimientos de calcio, así, de 1000 a 1200 mg/día en las menores de cincuenta años y de 1500 a $2000 \mathrm{mg} /$ día en las mayores de cincuenta años debido a que la absorción intestinal es de sólo $25 \%$. El calcio junto a la vitamina D y la calcitonina se requiere como sustrato para asegurar la conservación del hueso debido a la mayor remodelación que se efectúa durante esta época de la vida (17).

La ingesta de calcio por la alimentación es muy baja, aún en poblaciones de alto nivel económico. Un estudio en Estados Unidos (18) demostró que solo el 19,8\% de la población femenina tiene una ingesta de calcio mayor de $900 \mathrm{mg} /$ día (Véase tabla 7)

\section{Tabla 7}

\section{Consumo de calcio en la dieta y porcentaje de la} población femenina que lo ingiere

\begin{tabular}{|cc|}
\hline Ingesta $\mathrm{mg}$ /día & Población (\%) \\
\hline Entre 100 y 399 & 122,8 \\
Entre 400 y 699 & 43,6 \\
Entre 700 y 899 & 23,8 \\
Mayor de 900 & 19,8 \\
\hline
\end{tabular}

El mayor aporte de calcio por la dieta se obtiene principalmente de la leche y derivados; sin embargo, es frecuente ver la intolerancia y la aversión que tienen las personas adultas hacia estos productos (Véase tabla 8)

Una forma para estimar la ingesta diaria de calcio por persona es el cálculo de consumo diario de leche, sus derivados, y legumbres verdes; al resultado se le agregan $200 \mathrm{mg}$ que es el calcio aportado por el resto de la dieta (18).

Por lo anterior se puede apreciar que la dieta aporta escasa cantidad de calcio en pacientes con terapia de sustitución, excepto algunos alimentos específicos; por lo cual es necesario prescribir la terapia complementaria que, de acuerdo con el compuesto utilizado, aporta a la paciente una mayor o menor cantidad de calcio elemental. Así, el carbonato de calcio le aporta $40 \%$, el fosfato de calcio $23.3 \%$, el lactato de calcio $13 \%$ y el gluconato de calcio $9 \%$.

En colombia hay pocos compuestos que aseguren una complementación adecuada, uno de ellos es el calmin que tiene $500 \mathrm{mg}$ y el posture que libere $600 \mathrm{mg}$ de carbonato de calcio por cada comprimido, lo que equivale a $200 \mathrm{mg}$ de calcio elemental. La cal dolomítica, compuesta por carbonato de calcio y magnesio, puede presentar contaminación con metales pesados por lo cual su prescripción no es aconsejable.

La ingesta de calcio aumenta el efecto benéfico de la terapia estrogénica y del ejercicio, pues ayuda a disminuir el riesgo de osteoporosis. En caso de presentarse dicho riesgo es necesario administrar una ingesta de calcio de 1500 a $200 \mathrm{mg} /$ día contenidos entre la dieta y la medicación.

También, la disminución de la ingesta de alimentos que impiden la absorción de calcio disponible en el tracto intestinal. Dichos alimentos son: por ejemplo, el café en exceso, el cloruro de sodio, las porteínas y los carbohidratos:

No obstante, hay múltiples razones por las cuales no se aumenta la ingesta de calcio en la dieta:

1. La intolerancia a la leche, principalmente en esta época de la vida, debido a que en un $27 \%$ de las personas acentúan la deficiencia de la lactosa. Dichas personas pueden recurrir a la ingesta de yogur, el cual es mejor tolerado; además, éstas pueden usar citrato de sodio que aumenta la absorción del calcio, especialmente en personas 
Tabla 8

Contenido de calcio en los alimentos más utilizados

\begin{tabular}{|llc|}
\hline Alimento & \multicolumn{1}{c|}{ Cantidad } & Calcio (mg) \\
\hline Leche descremada & Una taza & 400 \\
Leche semidescremada & Una taza & 350 \\
Queso duro & $31 \mathrm{~g}$ & 200 \\
Queso crema & $31 \mathrm{~g}$ & 20 \\
Crema espesa & Una cucharada & 10 \\
Yogur & Una taza & 400 \\
Coliflor, apio y maíz & Media taza & $15^{\mathrm{a}}$ \\
pepino, lechuga y tomate & & $25^{\mathrm{b}}$ \\
Espárragos, col de brucelas, & Media taza & $175^{\mathrm{c}}$ \\
zanahoria y cebolla & & 200 \\
Repollo, nabo y bróculi & Media taza & \\
Sardinas y espinacas &
\end{tabular}

a Estos vegetales tienen contenido bajo de calcio

b Estos vegetales tienen contenido medio de calcio

c Estos vegetales tienen contenido alto de calcio

d En general, las carnes y los vegetales - excepto el bróculi, el repollo y el nabo- tienen muy poco contenido de calcio.

con acloridria, o pueden ingerir el calcio sobre las comidas o en la noche.

2. La disminución en la absorción de calcio en el tubo digestivo al ingerir vegetales rico en ácido fítico y oxálico, tal como las espinacas, o medicamentos que contengan aluminio, como los antiácidos, los glucocorticoides, la furosemida, las tetraciclinas y la isoniazida.

3. El aumento de la excreción de calcio debido a los alimentos ricos en proteínas; el cloruro de sodio en dosis mayor de $2000 \mathrm{mg} /$ día, es decir una cucharadita, el exceso de café, cinco pocillos; alcohol, cinco vasos; y cigarrillo. Este último también altera la retención del calcio en el hueso.

En conclusión, es importante que el médico proporcione una información muy clara a todas las pacientes para que tengan una nutrición adecuada en esta época de la vida.

A continuación se indican las sugerencias elaboradas por el Departamento de Agricultura en Estados Unidos para el manejo de las personas en edad avanzada:

1. Comer alimentos variados que comprenden los cuatro grupos siguientes: 1) frutas y verduras, 2) granos y cereales, 3) leche y derivados, y 4) proteínas.

2. Evitar alimentos con demasiada grasa, grasas saturadas y colesterol; sólo se deben ingerir en cantidades limitadas.

3. Comer alimentos con almidón y fibra en cantidades suficientes

4. Evitar comer demasiada azúcar

5. Evitar comer exagerada cantidad de cloruro de sodio

6. Moderar la ingestión de bebidas alcohólicas

7. Mantener el peso adecuado para cada persona

\section{Ejercicio}

Estudios prospectivos han demostrado que el mantener un estilo de vida que comprenda algún grado de actividad física, fundamentalmente con la realización de ejercicios dinámicos, se asocia a una reducción sustancial en la incidencia de enfermedad coronaria (19).

Estudios epidemiológicos demuestran un efecto cardio protector cuando se administra terapia de sustitución hormonal durante la menopausia; sin embargo, esta alternativa es poco útil si no se toman otras medidas complementarias tales como: realizar ejercicio físico, llevar una dieta bien balanceada, no fumar y controlar el peso corporal, la presión arterial y la glucemia.

La inactividad física tiende a provocar una serie de factores de riesgo que aumentan la incidencia de infarto del miocardio; uno de dichos factores es la hemoconcentración, la cual origina la elevación del hematócrito, la hemoglobina, el fibrinógeno, la viscosidad sanguínea y la adhesividad plaquetaria.

El ejercicio se asocia a una mayor actividad estrogénica, ya que aumenta el efecto osteoblástico; por ello se ha descrito una mayor cantidad de masa ósea en atletas, danzarinas y pesistas. Además del efecto sistemático, el ejercicio tiene un efecto local sobre la generación de masa ósea, pues, los huesos suelen desarrollarse según el esfuerzo al cual se someten. El ejercicio debe ser vigoroso y diversificado para que tenga un efecto osteogénico.

La realización de ejercicio físico: caminar, subir escalas y trotar entre treinta y sesenta minutos tres veces por semana con intensidad moderada, siempre y cuando no haya contraindicación, puede llevar un incremento significativo de la masa ósea; a la modificación de las lipoproteínas -disminuye los triglicéridos y aumenta el HDL-; a la reducción del peso corporal; y a la sensación de bienestar y dinamismo. Además, si el ejercicio se hace a la luz del día se obtiene el efecto positivo de los rayos del sol lo cual potencia la mayor síntesis de vitamina D. En ocasiones es necesaria la evaluación de un cardiólogo y una prueba de esfuerzo antes de iniciar cualquier ejercicio en personas sedentarias y obesas. 
De lo anterior se deduce que el manejo de la mujer en menopausia es integral y que el médico debe hacerla consciente de la necesidad de tener una vida útil para el tiempo de su existencia, lo cual se logra tratando de conservar su salud y aumentando la autoestima y la autonomía.

En conclusión, el médico necesita estar preparado para el manejo de la paciente con terapia hormonal debido a los siguientes motivos:

1. Aumento de la expectativa de la vida de la mujer, no sólo en los países desarrollados sino también en los que están en vía de desarrollo, lo cual hace que la mujer viva cerca de un tercio de su vida en menopausia.

2. Incremento en la población mundial de mujeres en estado de menopausia con su morbilidad asociada; para 1995 sobre una población mundial calculada en 2,8 billones de mujeres se espera un $50 \%$ de ellas con más de 49 años.

3. Escaso conocimiento e información que tiene la mujer acerca de los fenómenos presentes durante esta época de la vida, lo cual la lleva a suspender rápidamente la terapia hormonal prescrita por el médico, es así como el $50 \%$ de las mujeres en Estados Unidos y el $75 \%$ en otros países suspende el tratamiento a los seis meses, o menos, de su prescripción.

4. Poca información, preparación y motivación, así como el temor del cuerpo médico para prescribir terapia hormonal durante este período. Se calcula que únicamente $1,8 \%$ de la población mundial con déficit estrogénico recibe terapia hormonal, lo cual se convierte en un problema de salud pública.

\section{BIBLIOGRAFIA}

1. Sitruk-Ware R. Perimenopausia y postmenopausia. En: Medicina de la Reproducción, Ginecología Endocrinológica. Editores Mauvais Jarvis P., Sitruk-Ware R., Labrie F. Versión española Puig Menen A. Ediciones Toray Barcelona. 1985; 400-417.

2. Harlap S. The benefits and risks of hormone replacement therapy: An epidemiology over view.Am. J. Obstet. Gynecol. 1986; 166: 19861992.

3. Henderson BE., Paganini-Hill A., Ross RK. Decreased mortality in users of estrogen replacement therapy. Arch. Intern. Medic 1991; 151: 75-78.

4. Gambellr RD. Studies of endometrial and breast disease with hormone replacement therapy in the menopause. Edited John WW Studd, Whitehead Ml. Blackwell scientific publications Oxford. 1988; 247261.

5. Huppert LC. Tratamiento sustitutivo hormonal: Beneficios, riesgos, dosis. Clinic Médic N.Am. 1987; 1:27-41.

6. Thom MH. White PJ., Williams RM. et al. The prevention and treatment of endometrial disease in climateric women receibing oestrogen therapy. Lancet. 1979; 455-457.

7. Studd L., Magos A. Oestrogen therapy and endometrial pathology. In: Studd J. WW., Whitehead MI. The menopause Oxford. Blackwell scientific publications. 1988; 197-212.

8. Creasman WT. Estrogen replacement therapy: is previosly treated cancer a contraindication? Obstet. Gynecol. 1991; 77:308-312.

9. Steinberg KK., Thacker SB., Smith J. et al. A meta-analysis of the effect of estrogen replacement therapy on the risk of breast cancer. JAMA. 1991; 265: 1985-1990.

10. Sillero-Arenas M., Delgado-Rodríguez, M., Rodríguez-Canteros. Menopausal hormone replacement therapy and breast cancer: A meta-análisis. Obstet. Gynecol. 1992; 79: 286-294.
11. Cardozo LD. Routes of estrogen administration. In: studd J. W. W., Whitehead MI. The menopause Oxford. Blackwell scientific publications. 1988; 138-144.

12. Powers MS., Schenkel L., Darley PE. Pharamacokinetics and pharmacodynamics of transdermal dosage forms of $17 \mathrm{~B}$-estradiol: comparison with conventional oral estrogens used for hormone replacement. Am.J. Obstet. Gynecol. 1985; 152: 1099-1106.

13. Fahraeus L. The effects of estradiol on blood lipids and lipoproteins in postmenopausal women. Obstet. Gynecol. 72 (Suppl) 1988; 185 225.

14. Henderson, BE., Ross, RK., Lobo RA., Pike MC., Mack TM. Revaluation the role of progestogen therapy after the menopause. Fertil Steril 49 (Suppl): 1988; 95-155.

15. Hassager C., Christiansen C. Estrogen/Gestagen therapy changes soft tissue body composition in postmenopausal women. Metabolism 1989; 38: 662-665.

16. Avioli LV. Calcium the menopause and osteoporosis. In: Peck W. A. Trends and perspectives in the diagnosis and management of osteoporosis. New Jersey. Parthenon Publishing grarp. 1988; 59-65.

17. Sutnick MR. Nutrición, calcio, colesterol y calorías. Clinic Medic N Am 1987; 1: 117-128.

18. Notelovitz M. The influence of nutrition and exercise. In: Greenblatt R. B. A. modern approach to the perimenopausal year. New York. Walter de Grayter. 1986; 109-115.

19. Caraballo D. Ejercicio y climaterio presentado en Primer Simposio Nacional de Menopausia. Medellín. Imprenta Universidad de Antioquia. 1992; 87-94. 